\title{
Sexo ambiguo con constitución cromosómica
}

$$
45, \mathrm{X} / 45, \mathrm{X}, \mathrm{t}(\mathrm{Y} ; \mathbf{1 4})
$$

Dra. Carmen Noziglia del N ${ }^{1}:$ T.M. M. Angélica Alijende R. 2:T.M. M. Virginia Carvajal D.2; Dr. Walter Radjigán V. ${ }^{3}$; Dr. Raúl González A.4; Dr. Jorge Ruiz K. ${ }^{5}$; Dr. Ives Lacassie S. ${ }^{2}$

\section{Ambiguous genitalia and $45, X / 45, X, 1(Y ; 14)$ chromosomal constitution}

\begin{abstract}
We report a patient evaluated in the newborn period because of ambiguous genitalia who presented normal hormonal levels and a $45, X / 45, X, t(Y ; 14)$ karyotype. During the surgical repair of an inguinal hernia, Mullerian derivates and gonads of misleading aspect werc demonstrated. The histopathological study showed a streak gonad and an embriological teste. With the diagnosis of mixed gonadal dysgenesis, the teste was removed. The importance of cytogenetic, hormonal and histopathological studics in the djagnostic evaluation of patients with ambiguous genitalia is stressed. The hypothesis of a half chromalid transiocation could explain the structural mosaicism found.

(Key words: Ambiguous genitalia, chromosomal abnormalitics, mosaicism, half chromatid translocation, cytogenetics).
\end{abstract}

La incidencia de anormalidades cromosómicas mayores tanto numericas como estructurales es de 5,72 por 1.000 recién nacidos vivos 1 . La translocación del cromosoma $Y$ a uno de Ios autosomas tiene una incidencia de aproximada. mente 1 en 2.000 recién nacidos ${ }^{2-4}$. Aunque hay casos descritos en pacientes con fenotipos alterados, estas translocaciones constituyen un hallazgo citogenético en personas sin manifestaciones físicas o mentales. Tampoco se ha descrito aumento de la incidencia de infertilidad o de abortos espontáneos en las familias de los probando $s^{1,3,4}$. La presencia de mosaicismos que comprometen los cromosomas sexuales y producen disgenesia gonadal o sexo ambiguo const $\mathrm{i}$ tuye una patología cromosómica algo más infrecuent $e^{1}$.

1. Servicio de pediatría, Hospital Naval, Valparaíso y Hospital Gustavo F'ricke, Viña del Mar.

2. Unidad de genćtica, INTA, Universidad de Chile.

3. Servicio de cirugía infantił, Hospital Naval, Valparaiso.

4. Servicio de anatomia patológica, Hospital Gustavo Fricke, Viña del Mar.

5. Laboratorio de endocrinología, Hospital Naval, Valparaíso.

* Trabajo presentado en la XVII Reunión Anual de la Sociedad de Genética de Chile, Santiago, 1984.
En este trabajo se describe un niño que fue evaluado de recién nacido por presentar sexo ambiguo, cuyo estudio citogenético reveló una constitución cromosómica $45, \mathrm{X} / 45, \mathrm{X}, \mathrm{t}$ (Y; 14) y en que el estudio histopatológico de góna. das demostró disgenesia gonadal mixta.

\section{Casso clínico}

Recién nacido, de padre (23 años) y madre (22 años), sanos, no consanguíneos y cuyo único antecedente familiar es el de un tío paterno con sindrome de Down. Producto del primer emba. razo, deseado, controlado, sin complicaciones. Parto eutócico, a las 42 semanas de gestación (7/1/84), peso $2.660 \mathrm{~g}$ (P 10 Lubchenco), talla $46,5 \mathrm{~cm}$ (sobre $P 10$ ), circunferencia craneana (CC) $33 \mathrm{~cm}$ (bajo P 25) y Apgar 9 al minuto. En Jos genitales (Eig. 1), se observaba la presencia de labios mayores sin gónadas palpables (A,B), clitoris hipertrófico (A-D) y orificio uretral perineal cón canal abierto hasta el extremo libre del clítoris (C). La evaluación endocrinológica se realizó a la semana de vida: 17 cetoesteroides urinarios (17 Ks): $0,3 \mathrm{mg} \mathrm{x} 24 \mathrm{~h}$ y $0,4 \mathrm{mg} \mathrm{x} 24 \mathrm{~h}$; 17 hidroxiesteroides urinarios $(170 \mathrm{H}): 2.4 \mathrm{y}$ $0,7 \mathrm{mg} \times 24 \mathrm{~h}$; Pregnantriol urinario 0: Dehidroepiandrosterona urinaria $0,1 \mathrm{mg} \times 24 \mathrm{~h}$; Progesterona plasmática: $0,35 \mathrm{mg} \times \mathrm{ml}$; Cortisol plasmático (A.M.): $16,3 \mu \mathrm{g} \%$; Testosterona plasmátj- 


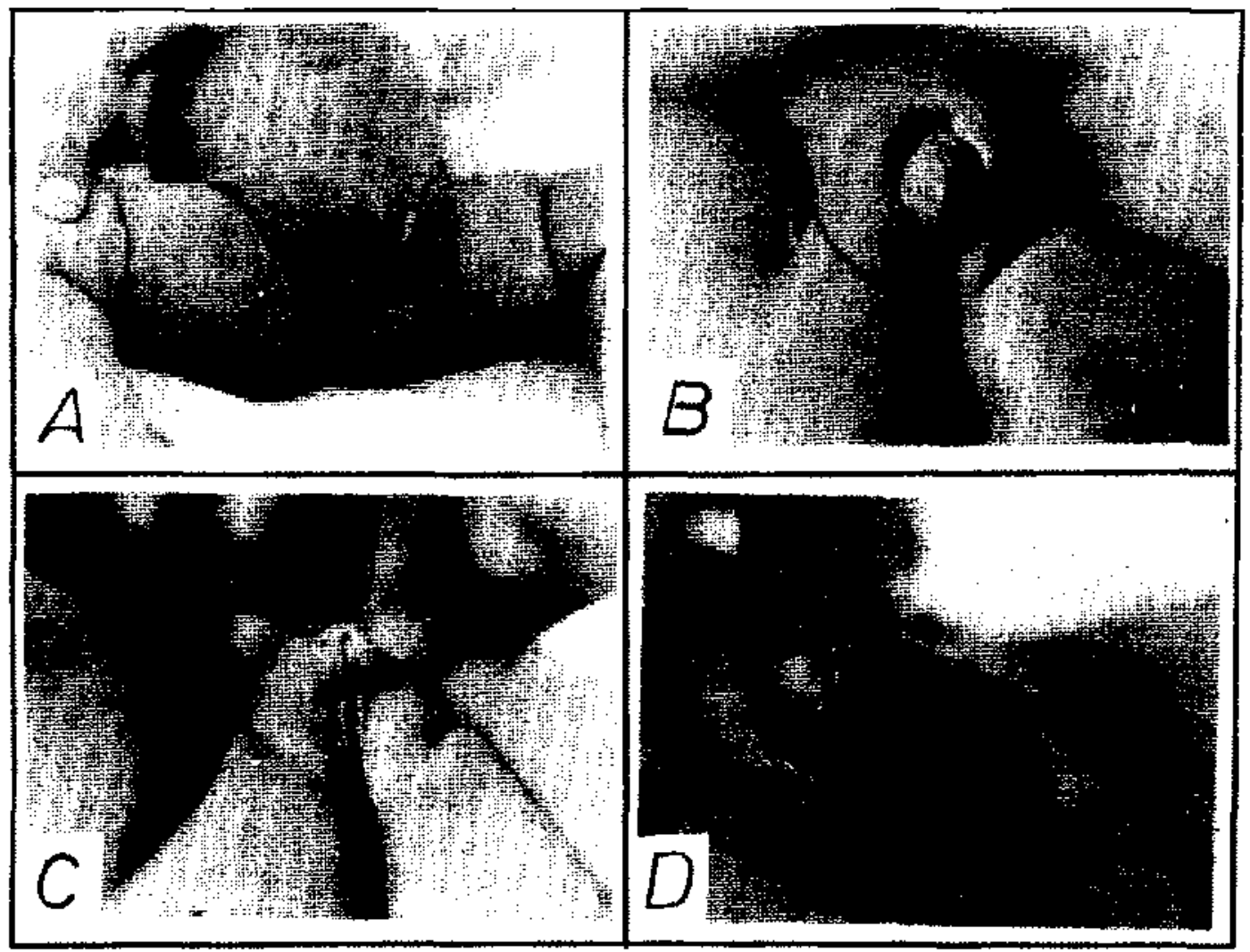

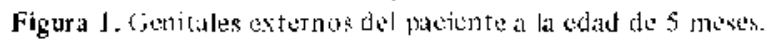

ca: $50 \mathrm{ng} / \mathrm{dl}$ ); pielogralía y cistografía normales. Cromatina nuelear negativa. El cariograma con téenica de bandeo GTC, Q y CGB ${ }^{5}$ demostró una constitución cromosómea $45, \mathrm{X}$ ! 45, X, t $(\mathrm{Y}$; 14) (Fig. 2). cada lined en el 50\% de las mitosis.

Durante los primeros 11 meses de vida el ritmo de srecimicnto y el desarrollo psicomotor fueron normales. Fn el control de los 5 meses ademas de las alteraciones descritas en el periodo de recién nacido - llanó la atención la presencja de hernia inguinal izquierdia. Antes de proceder a su reparación quirúrgica (20/6/84), se exploró, bajo anestesia general, el orificio posterjor de la uretra, demostrando la existencia de una vagina de $4 \mathrm{~cm}$ de profundidad y cseaso diámetro, por lo que sc amplió su apertura al exterior. Duratue la reparación de la hernia se encontró un pequeño útero con dos trompas en posición normal. Contigua a la trompa izquicra existía una gúnada que parecia ovar io normal. En el ligamento ancho del mismo lado existía otra masa que se interpretó como testículo atrófico por to que fue extrpada. En la exploración del lado derecho no se detectó gónada. Se tomaron muestras de tejido de ambas trompas y de la gónada, cuyo estudio histopatológico mostró que ambas trompas ulerijas eran histológicamente normales (t.ig. $3-\hat{A}$ ): pero la mas que macroscópicamente parecía uvario nornal presentaba capa albuginca definida, túbulos seminíferos embrionarios, poco dexirrollatos, reducción del diametro lubular medio indice de lertilidad $50 \%$ $y$ predominio de células de Sertoli innaduras. El intersticio incluia escasas células de Leydig (Fig. 3 B). En ur sector de la mest ta se observo una estructura con caracteres de epidídimo, con ductus, machos de ellos dilatidos. $y$ epitelio estratilicado alto (Fig. 3-C). Se concluyó que se trataba del tejido testicular de tipo cmbrionario con epidídimo. La masa del ligamento ancho que macroscópicamente parecia un testiculo disgenético presentaha estroma fusocelular denso, cordones epiteliales primitivos alternando con zonas ricumente vascularizadas y zonas de tibrosis densa (Fig. 3 D). concluyéndose que correspondía a unia gónada veteada.

\section{DISCUSION}

Lá conjunción de dos alteraciones cromosómicas. como translocalión $(Y ; 14)$ en mosaico en un paciente $45 . X$ es poco frecuente e interesante. Li translocación de un cromosona $Y$ a un autosoma parecicra ser un hallazgo sin mayor tascen- 


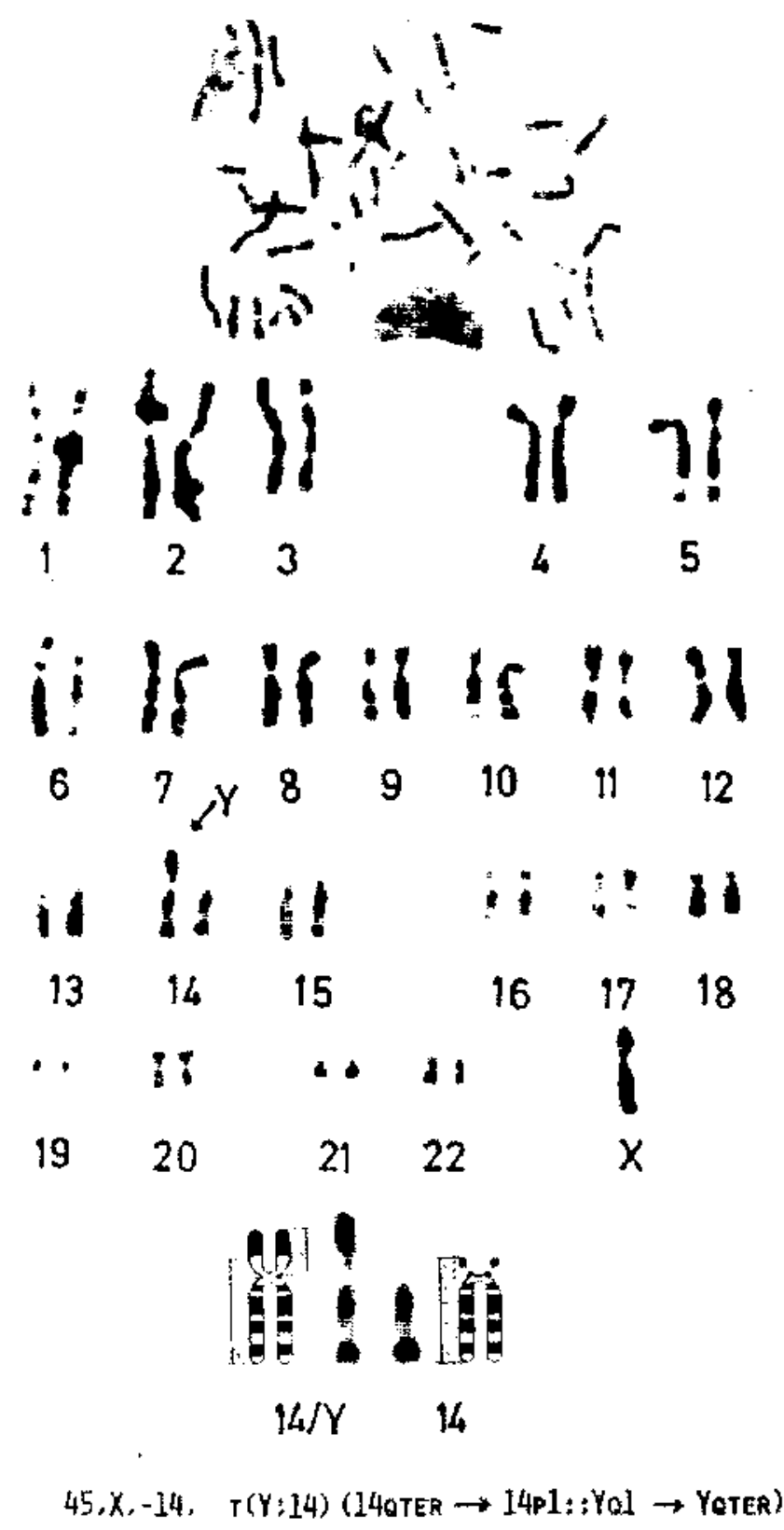

Figura 2. Cariotipo con bandeo GTG de la línea celular 45.X, t (Y:14) presente en el $50 \%$ de las mitosis. La otra línea celular era 45.X.

dencia de acuerdo con un estudio en poblaciones no seleccionadas ${ }^{3}$. Las translocaciones descritas con más frecuencia son $\mathrm{Y} / 15$ e $\mathrm{Y} / 22$, aunque también se han encontrado transtocaciones $Y / 5$, $\mathrm{Y} / 7, \mathrm{Y} / 13$ e $\mathrm{Y} / 2 \mathrm{I}^{3}$. Cuando el cromosoma $\mathrm{Y}$ se transloca a] $X$, los sujetos pueden ser de sexo masculino o femenino, dependiendo de la porción del cromosoma $\mathrm{Y}$ comprometido ${ }^{6}$. Los determinantes testiculares parecen estar localizados en el brazo corto (Yp), cerca del centrómero ${ }^{7} \mathrm{~s}$.
Se sabe que estos determinantes son responsables de la diferenciación de las gónadas embrionarias indiferenciadas en testículos. Esta parece ser la única función genética del cromosoma $Y$, ya que la diferenciación masculina se efectúa bajo la influencia de andrógenos secretados por los testículos ${ }^{9}$. Si sólo existe $\mathrm{Yq}$, los pacientes son de sexo femenino y la translocación puede ser trasmitida a hijos hombres o mujeres ${ }^{6}$.

El mosaicismo consiste en la presencia simul- 


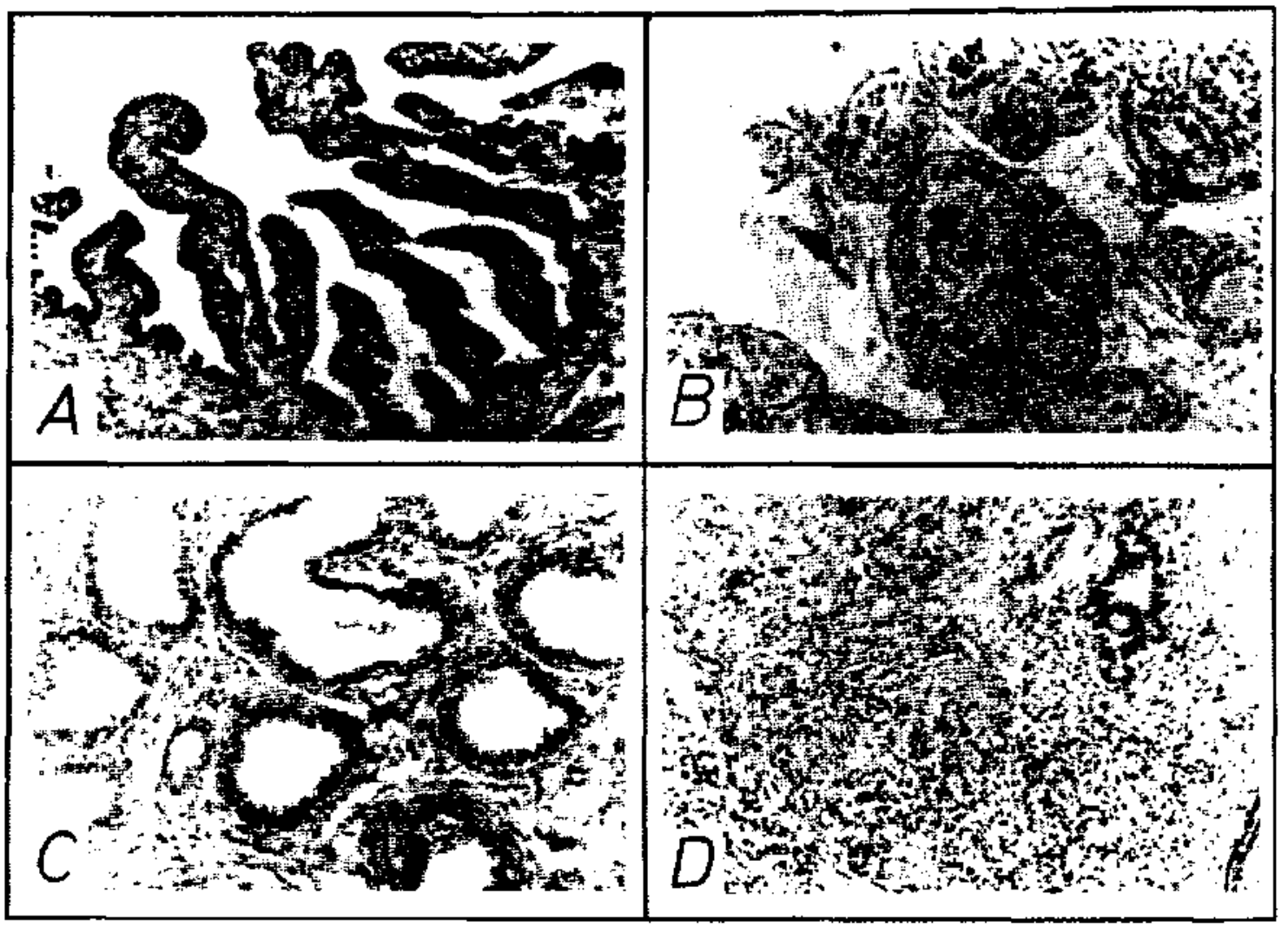

Figura 3. Hallazgos histopatológicos; A. Trompa normal; B. testiculo; C. epididimo; D. gónada veteada.

tánea de dos o más lineas celulares diferentes en un indjviduo. Tradicionalmente los mosaicos se han explicado como resultantes de no disyunción o retardo de la anafase mitótica luego que un espermio normal fecunda a un óvulo tambièn normal. Sin embargo, utilizando marcadores cromosómicos, se ha demostrado recientemente que un porcentaje importante de los mosaicos de sindrome de Down provienen de una no disyun. ción mejótica ${ }^{10}$. La forma más corriente de mosaicismo asociado a disgenesia gonadal es $45, X / 46, X^{7}$. Los individuos que presentan una línea celular $45, X, y$ por lo menos una que contenga un cromosoma. Y, pueden manifestar toda una gama de fenotipos: genitales externos femeninos, ambiguos, masculinos con criptorquidea o hipospadia $y$ ocasionalmente masculinos casi normales ${ }^{7}$. Otros mosaicos más raros son $45, \mathrm{X} / 47, \mathrm{XYY}$ y $45, \mathrm{X} / 46, \mathrm{XY}$ que aparentemente están asociados al mismo espectro fenotípico ${ }^{7}$. También han sido descritos mosaicos $X /$ $\mathrm{XX} / \mathrm{XY}, \mathrm{X} / \mathrm{XXXY}, \mathrm{X} / \mathrm{Xiso} \mathrm{YY}, \mathrm{X} / \mathrm{XY}$ / $\mathrm{XXX}^{11,12}, \mathrm{X} / \mathrm{XXX}, \mathrm{XX} / \mathrm{XX}-\mathrm{X} / \mathrm{XYP}-, \mathrm{X}$ / XXXY, X / XYYY ${ }^{3}$, que pueden mostrar estigmas del sindrome de Turner o no tener anomalías somáticas.

La mayoría de los pacientes $45, \mathrm{X} / 46, \mathrm{XY}$ con genitales externos femeninos o ambiguos posee derivados Mullerianos ${ }^{7}$. Las gónadas de los pacientes $45, \mathrm{X} / 46, \mathrm{XY}$ pueden ser testiculos disgenéticos o gónadas "veteadas". Las gónadas "veteadas" no pueden distinguirse histológica. mente de las encontradas en personas con disgenesia gonadal $45, X^{7}$. En las disgenesias gonadales mixtas también pueden encontrarse ovotestes y ovario. En cuanto al ductus que acompaña a las gónadas: siempre al lado de un ovario hay una trompa; junto a un testículo generalmente, hay deferente y epididimo. pero se han descrito casos en que se ha encontrado una trompa y en éstos la biopsia del testículo ha demostrado que es inmaduro; adyacente a un ovotestes puede haber una trompa o un deferente ${ }^{\mathbf{l}}$. La apariencia macros. cópica del ducto suele ser equívoca. No es raro que una aparente trompa hipoplástica resulte ser un conducto deferente en el examen microscópico. Lo contrario no sucede $y$ siempre que macroscópicamente se ve un deferente, el examen microscópico lo confirma. Además, siempre que hay deferente también hay epidídj$\mathrm{mo}^{13}$

La posibilidad de mayor riesgo de no disyunción en portadores de translocación Y/autosoma aún no está resuelta ${ }^{3}$. Existen casos, posible- 
mente excepcionales, en que portadores de translocaciones de este tipo han tenido hijos con la translocación y además una trisomía $21, \mathrm{XXY}$ o una monosomia $X^{3}$. Por otra parte se han descrito familias que, además de translocación entre autosomas involucrando el 21 , han presentado mosaicismo de los cromosomas sexuales. Más aún, otros miembros de una de estas familias sólo presentabán dicho mosaicismo ${ }^{14}$. Aunque aún hay controversa, cada dia existe más evidencia de que las translocaciones involucran mayor riesgo de no disyunción ${ }^{15}$. En nuestro caso, la translocación cromosómica que compromete al cromosoma $\mathrm{Y}$ fue confirmada mediante bandeo GTG (bandeo $G$ con Tripsina y Giemsa) y bandeos $\mathrm{C}$ y $\mathrm{Q}$ que permiten reconocer con más facilidad el cromosoma $Y$ debido a la fluorescen. cia característica de su brazo largo con la quinacrina que, aunque puede estar ausente en un bajo porcentaje de hombres normales, se detectó en el probando. Dado que la mayor parte del cromosoma $Y$ estaria translocada al cromosoma 14 algún determinante testicular estaría presente.

El estudio cromosómico de ambos padres fue normal por to que las alteraciones del probando sucedieron "de novo". El hallazgo de un tío paterno con trisomía 21 en la familia apoyaría la posible existencia de factores genéticos familiares para no disyunción.

La doble alteración cromosómica, se puede explicar de manera similar a los mosaicos estruc. turales ${ }^{16}$; si durante la primera fase de síntesis del DNA en la espermatogénesis se produce una rotura en media cromátide del cromosoma $Y$, con unión a un cromosoma 14, al final de la espermatogénesis habría 3 espermios con dotación haploide normal y uno con 22 cromosomas (t $Y / 14)$. Al fecundar, este espermio, un óvulo normal se produciria un cigoto $45, X$ con una cromátide del $Y$ translocada a un cromosoma 14. En la primera división del huevo, una célula continuaria con la translocación mientras la otra tendría una constitución $45, \mathrm{X}$, obteniéndose el carotipo final $45, X / 45 . X, t(Y ; 14)$. Una discusión detallada de este mecanismo es motivo de otro trabajo ${ }^{17}$.

A pesar de que el sexo ambiguo fue sospecha. do por el pediatra en periodo neonatal y se comenzó el estudio de esta anomalía de inmediato, los padres le asignaton sexo femenino debido a las características de los genitales externos. Cuando se obtuvo el resultado del cariograma, se discutió la dificultad de asignarle sexo masculino por el tamaño del clítoris y lo difícil que sería la reconstrucción quirúrgica de la uretra peneana. La existencia de una vagina larga, terminó por decidir completamente la asignación de sexo femenino, lo que se vió reforzado por el hallazgo de útero y trompas de ubicación normal.

Los valores normales de testosterona de nuestro laboratorio, que utiliza la técnica de RIA de Forest $^{18}$ modificada, son 0 a $100 \mathrm{ng} x \mathrm{dl}$ en mujeres y 300 a $1.000 \mathrm{ng} x$ al en hombres, no teniendo valores normales para niños. Los valores normales para recién nacidos con la técnica de Forest ${ }^{17}$ son $68,1 \pm 59,5 \mathrm{ng} x$ dl en varones y 12 $\pm 6,2 \mathrm{ng} x \mathrm{dl}$ en mujeres. Ellos bajan a $6,6 \mathrm{ng} \%$ en ambos sexos a la edad de 10 meses y se mantienen hasta la pubertad. La concentración de $50 \mathrm{ng}$ $x \mathrm{~d} l$ encontrado en nuestro paciente a los 4 meses de vida, parece estar de acuerdo con la presencia de un testiculo ${ }^{18}$ debido a la existencia del cromosoma $\mathrm{Y}$ translocado.

En pacientes con hermafroditismo verdadero operados suelen haber errores en la interpretación de los hallazgos quirúrgicos de gónadas y ductos. Esto sucede especialmente en la apreciación de aparentes trompas que histológicamente resultan ser conductos deferentes. Por esta razón en la intervención se tomaron biopsias de trompas y de gónadas, cuyo diagnóstico, en efecto, fue distinto al macroscópico confirmán. dose que, en este tipo de pacientes, es fundamen. tal el estudio histopatológico.

En los pacientes con disgenesia gonadal en que el cariograma, demuestra la existencia de un cromosoma $Y$, la conducta debería ser la extirpación de las gónadas en el momento del diagnóstico, ya que en 15 a $20 \%$ de los casos se desarrollan gonadoblastomas o disgerminomas, ocasionalmente durante las dos primeras décadas de la vida ${ }^{7}$. Los gonadoblastomas son tumores constituidos por elementos celulares embriona. rios, germinales $y$ no germinales (derivados del estroma de los cordones sexuales); en sí no son malignos pero lo son potencialmente o pueden estar asociados con disgerminomas $\mathrm{u}$ otros tumores malignos de células germinales, ocurriendo casi exclusivamente en gónadas disgenétj$\operatorname{cas}^{7,19,20}$. Los disgerminomas son los neoplasmas malignos mas frecuentes en pacientes con disgenesia gonadal, con frecuencia representan crecimientos neoplásticos del componente de células germinales (células de tipo granulosa o de Sertoli) de un gonadoblastoma ${ }^{19,20}$. También pueden desarrollar coriocarcinoma (o caorioepitelioma) o carcinoma embrionario.

Las gónadas veteadas de personas $45, \mathrm{X} /$ $46, \mathrm{XY}$, que por lo general no pueden distinguirse histológicamente de las de otras con disgenesia gonadal $45, X$, suelen presentar gonadoblastomas y disgerminomas ${ }^{17}$, a diferencia de los pacientes con síndrome de Turner que tienen poco riesgo.

Dado que en la primera intervención no se extirpó la gónada que macroscópicamente parecía oyario normal y posteriormente resultó ser un 
testículo, se realizó una nueva intervención a los 10 meses de vida para extraerlo, por su potencial maligno y porque su producción de testosterona acarrearía virilización en una persona a la que se la hab ía asignado sexo femenino.

La discreta clitoromegalia y leve fusión labial posterior observada sugieren alguna virilización ocurrida durante la vida fetal. Sin embargo, por la posición del meato uretral ésta debe haber sido escasa. Durante los meses de seguimiento de esta paciente se pudo observar un aumento de la clitoromegalja que aparentemente $s$ detuvo con la extirpación del testículo a los 10 meses de vida.

Este caso tiene interés por la concomitancia de dos alteraciones cromosómicas en un mismo paciente y porque permite enfatizar los posibles errores en la interpretación de los hallasgos macroscópicos y la necesidad de contar con estudios de laboratorio, cromosómicos, hormonales $\mathrm{e}$ histológicos, en este tipo de afecciones.

\section{RESUMEN}

Se presenta un paciente evaluado en el PRN por presentar sexo ambiguo cuyo estudio hormonal fue normal pero la evaluación citogenética demostró la existencia de una translocación (Y;14) en mosaico: 45,X/45,X,t $(Y ; 14)$, posible de explicar por una translocación de media cromátide. Durante una hernioplastía se demostró la existencia de derivados mullerianos y dos gónadas de impresión equívoca que. histológicamente, correspondieron a testículo embrionario y a gónada veteada. Con el diagnóstico de disgenesia gonadal mixta el testículo fue ulteriormente extirpado. Se enfatiza la importancia de los estudios citogenéticos, hormonales e histopatológicos en la evaluación diagnóstica de pacientes con sexo ambiguo.

\section{AGRADECIMIENTOS}

Agradecemos a la Dra. Fanny Corfes su Aesintereseda colaboración en la preparación del manuscrito.

\section{REFERENCIAS}

1. Friedrich, U., Nielsen, $J_{\text {.: }}$ Chromosome studies in 5.049 consecutive newborn clildren. Clin. Genet. 4: $333,1973$.
2. Schmid, M., Schmidtke, J., Kruse, K., Tolkdorf, M.: Characterization of a $Y / 15$ translocation by banding methods, distamycin. $A$ treatment of lymphocytes and DNA restriction endonuclease analysis. Clin. Genct. 24: 234, 1983

3. Vielsen, J., Rasmussen, $K,:$ Y/Au tosomal translocation. Clín. Genet. 9: 609.1976.

4. Nieisen, J., Friedrich, U., Hreidorsson, A.B., Noel, $B$. Quack, B., Motter, $J_{\text {: }}$ Brilliantly fluorescing colarged short arms D or G. Lancet 1: 1049, 1974.

5. Dutrilaux, E. Couturier, $J$.: La practixue de lanalyse chromosomique. Techniques de Latrorator ies. Maswon, l'arís, $\mathbf{9 8 1 .}$

6. Zuffarai, O. Maraschio, P., Lo Curto. F., Muller. U., Giorola, A., Peroti, L.: The role of Yp in sex determination: New Fvidence from X/Y Translocation. Am. J. Med. Genet. 12: 175, 1982.

7. Simpson, J.L.: Disorders of sexual differentiation. Etiology and Clinical delineation. Academic Press. New York, 1976.

8. McKustek, V.A.: The Human Gene Map 1 Deecnber 1984. Clin. Genet, 27: 207, 1985.

9. Cossorla, F.G. Emanuel, B.S. Parks, J.S. Wu, C.H. Wheeler, JE., Tenore, A.: Cytogenetic and endocrine findings in a female whth $45, X, t(Y ; I B)$ (p 11 : p11). Clin. Genet. 19: 312,1981.

10. Nikawa, N., Kajii, T.: The origin of mosic Down synclome: Four cases with chromosome narkers. Am. J. Hum. Genet. 36: 123, 1984.

11. Josso, N., De Grouchy, J., Nezelof, C., Frezal, J., Jammet. M.L., Lamy, M.: Mixed gonadal dysgenesis. Am. J. Dis. Child. 112: $531,1966$.

12. Gardner, L.t.: Endocrinc and Genetic discase of childhood and adolescence. W.B. Saunders Co. Philadelphia, 2nd. Ed. 1975.

13. Van Niekerk, W.A.: True IIemphroditism. Harper and R ow Publishers Inc., Mary Iand, 1974.

14. Smith, A., Eltiott. B.: Occutrence of sex chromosome moxajeism and translocation Down's syndrome in the same family. Clin. Genet. 17: 341, 1980.

15. Curotto, B., Secbach, Ch., Lacassie, Y.: Alteraciones cromosómicas ostructurales parentales y sindr $O$ me de Down. Arch. Biol. Med. Exp. 14: 50 (A). 1981.

16. Cantú, J.M., Rivas, F., Rivera, H., Ruiz, C.: The prezygotic origin of structural mosaicism. Ann. Genét. 28, 2: 73, 1985 .

17. Alliende, M.A., Cortés, F., Noziglia, C. Carrajal, M. $V$. Lacassie, $\mathrm{Y}^{\prime}:$ Mixcd gonadal dy genesid with $45, X ; 45, X, r(Y ; 14)$ explainabled by a prezygotic (meiotic) half chromatid translocation (Observaciones no publicadas).

18. Forest, M.G., Calhiard, A.M., Berkand, J.A.: Total and unbound testostcrone levels in the newborn and in normal and hypogonadal children: lise of a sensitive radioinmunoassay for lestostcrone. J. Clin. Eudocrinol Metab. 36: 1132, 1973.

19. Galloger, H.S., Lewis, R.P.: Sequential gonadoblastoma and choriocarinoma. Obstet Gynecol. 41: $123,1973$.

20. Tolerman, Ai, Musinga, W.T., Knipers, T.: Dy ggerminoma. Obstet. Gynecel. 41: 13?, 1973. 